\title{
Archéopages
}

Archéopages

Archéologie et société

$44 \mid 2017$

Terrains vagues

\section{Espaces vides et espaces bâtis dans la ville antique de Cahors-Divona}

Liens avec la topographie et évolution durant le Moyen Âge

Cahors-Divona. Wastelands during Roman antiquity

Cahors-Divona. Terrenos baldíos durante la Antigüedad romana

\section{Didier Rigal}

\section{OpenEdition}

Journals

Édition électronique

URL : https://journals.openedition.org/archeopages/1452

DOI : 10.4000/archeopages. 1452

ISSN : 2269-9872

Éditeur

INRAP - Institut national de recherches archéologiques préventives

Édition imprimée

Date de publication : 1 juin 2017

Pagination : 16-19

ISSN : 1622-8545

\section{Référence électronique}

Didier Rigal, «Espaces vides et espaces bâtis dans la ville antique de Cahors-Divona », Archéopages [En ligne], 44 | 2017, mis en ligne le 01 juillet 2019, consulté le 02 juin 2021. URL : http://

journals.openedition.org/archeopages/1452; DOI : https://doi.org/10.4000/archeopages.1452 


\section{Espaces vides et espaces bâtis dans la ville antique de Cahors-Divona \\ Liens avec la topographie et évolution durant le Moyen Âge}

Didier Rigal Inrap, UMR 5608, «Traces»
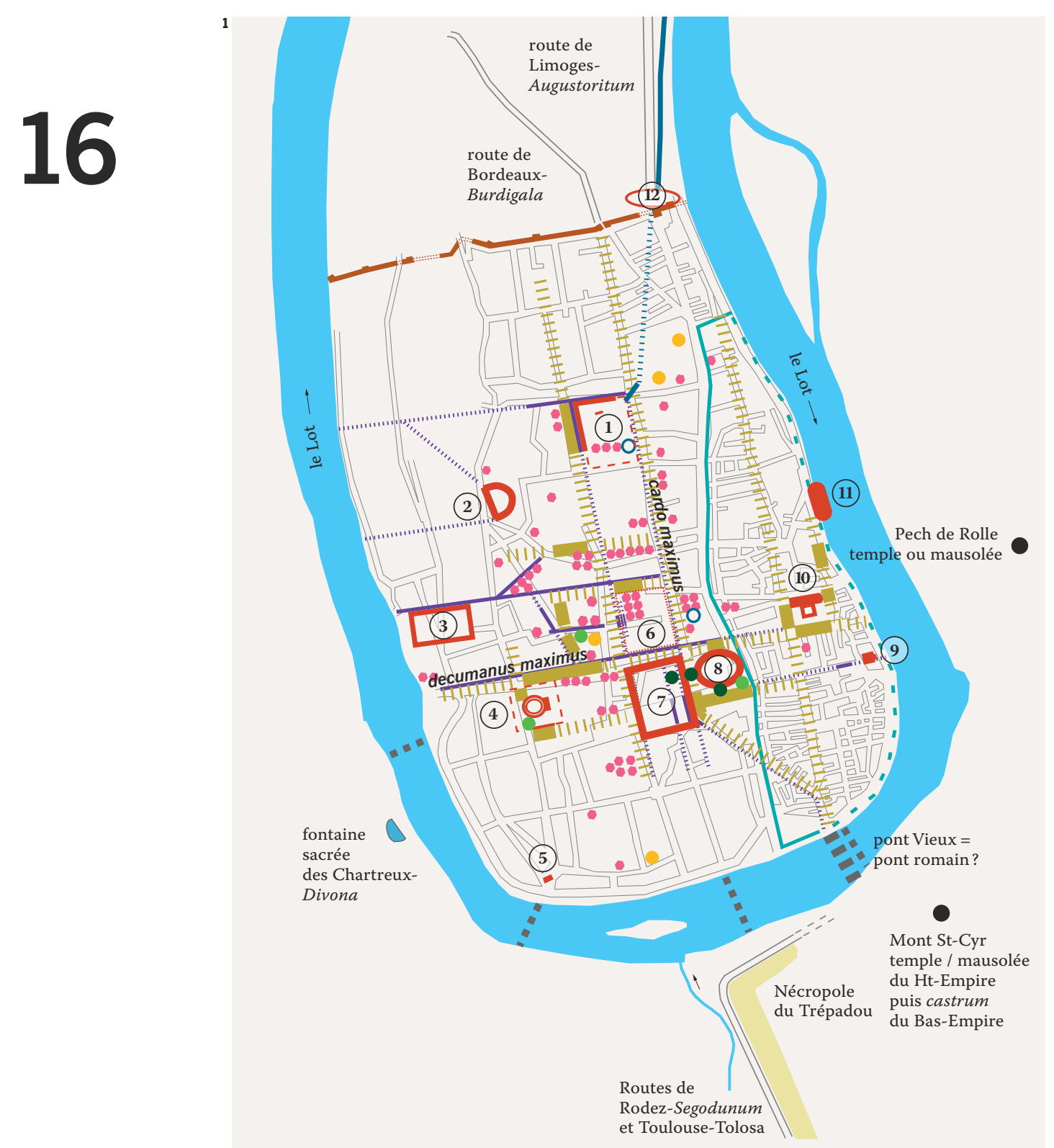

- aqueduc

II pont

- - gué (médiéval)

voirie

— égoût

○ puits

- mosaique

- artisanat terre cuite

- artisanat métallurgie

- artisanat chaux

— enceinte du Bas-Empire/viI ${ }^{\mathrm{e}}$.

- enceinte $\mathrm{XIII}^{\mathrm{e}}{ }^{\mathrm{X}} \mathrm{XIV}^{\mathrm{e}} \mathrm{s}$.

1. thermes publics

2. théâtre

3. schola

4. temple

5. tour des Chanoines

6. forum?

7. forum

8. amphithéâtre

9. église St. Urcisse

10. cathédrale

11. port?

12. castellum?

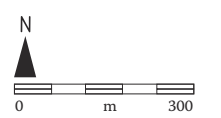

1. Principaux sites antiques répertoriés et éléments majeurs de la ville médiévale sur fond cadastral actuel. 
De création ex-nihilo, Cahors-Divona est fondée à la fin du règne d'Auguste, au moment du rattachement de son peuple à l'Aquitaine primitive, sur une surface dégagée de 210 ha. Le choix d'implantation est l'intérieur d'un méandre du Lot, rivière bordée de collines abruptes et de falaises, reliefs qui limitent l'expansion de la ville. On suppose que ses nouveaux habitants sont ceux transférés, au moins pour partie, depuis les oppida cadurques environnants (Murcens, Saint-Crépin, l'Impernal...) sur lesquels les traces de présence humaine disparaissent à la fin de l'indépendance. Au cœur de son territoire, la civitas gallo-romaine des Cadurques est également positionnée à un carrefour de routes la situant, avec des relais intermédiaires, à une centaine de kilomètres des chefs-lieux de cité voisins : au nord-ouest Périgueux-Vesunna, à l'ouest Agen-Aginnum, à l'est Rodez-Segodunum et, en direction du sud, Toulouse-Tolosa. Resserré au nord, à l'emplacement qu'occuperont les fortifications médiévales des XIII ${ }^{\mathrm{e}}-\mathrm{XIV}^{\mathrm{e}}$ siècles, l'isthme présente un pendage très marqué dans sa plus grande longueur. C'est ainsi qu'il domine de près de $30 \mathrm{~m}$ le Lot dans la partie nord, tandis qu'au sud, le niveau de la rivière se confondait avec les berges avant la réalisation des quais à la fin du XIX ${ }^{\mathrm{e}}$ siècle. En dépit de ces travaux, on assiste régulièrement, encore de nos jours, à des crues du Lot dans ses parties basses, au sud et à l'ouest.

Sur cet espace contraint par des caractéristiques morphologiques fortes, il nous a semblé particulièrement intéressant d'initier la réflexion sur le rapport entre les espaces bâtis et ceux laissés vides. Cette première ébauche se fonde sur les opérations archéologiques menées dans la ville entre 1990 et $2006^{\mathbf{1}}$.

\section{Les monuments et l'habitat}

Si quelques monuments publics étaient connus anciennement, l'interprétation de leur fonction a été revue, pour certains, et leur liste a été fortement augmentée par les fouilles programmées, sur l'aqueduc, puis préventives, menées par l'Afan puis l'Inrap qui ont permis, notamment, de découvrir le temple monumental et son portique (Rigal, 2004, 2008a), l'amphithéâtre, le forum (Rigal, 2008b), la probable schola (Grimbert, 2011) et le castellum du BasEmpire (Boudartchouk, Rigal, 2013). Cet ambitieux programme architectural de la capitale des Cadurques est mis en place en moins d'un siècle. Il débute autour du changement d'ère avec l'aqueduc (Rigal, 2011), et prend fin avec l'amphithéâtre deux décennies avant la fin du $\mathrm{I}^{\text {er }}$ siècle de notre ère [ill. 1] (Rigal, 2016, 2017). La plupart de ces monuments (la fontaine de Divona, le temple, le portique, le forum et l'amphithéâtre) ont la particularité de former un alignement continu d'est en ouest constituant une exceptionnelle scénographie monumentale faisant face aux routes de Tolosa et de Lugdunum.
Le choix d'implantation du castellum, des thermes et du théâtre repose sur l'exploitation de caractères topographiques favorables (présence du relief rocheux favorisant l'acoustique et l'entaille des gradins pour le théâtre, par exemple).

L'habitat se développe surtout au nord de cet axe monumental, sur 125 ha. Il est connu par près de 80 pavements mosaïqués qui sont bien souvent les uniques témoignages des domus, aucune d'elles n'ayant été fouillée à ce jour. Cet état de fait trouve en partie son origine dans le fait que la majeure partie du Cahors médiéval est sanctuarisé et donc peu susceptible de faire l'objet de prescriptions archéologiques.

\section{Les zones vides}

Au sud, l'espace compris entre la façade monumentale et les berges de la rivière couvre $400 \mathrm{~m} \times 1000 \mathrm{~m}$, soit 40 ha. Les observations y ont été peu nombreuses, mais y ont été repérés principalement des épandages de matériaux de démolition et des ateliers de production de terre cuite en bordure de la rivière ; deux types d'usages habituels à la périphérie d'une ville antique, en zone inondable. La volonté de stabiliser ces terrains limoneux instables, fréquemment sujets aux débordements de la rivière, est évidente. Il s'agit d'un terrain non cultivé, non arboré, non bâti ${ }^{2}$, sinon de structures légères, mais utilisé et fréquenté. Sans doute faudrait-il rechercher sur les berges un port, ou à défaut des appontements, permettant le déchargement des marchandises transportées sur la rivière, ainsi que des entrepôts dont la présence semble incontournable, mais qui restent à découvrir.

Nous ignorons encore à quelles utilisations était vouée la grande surface au nord de la ville. On pourrait s'attendre à ce que ces zones hors la ville, en bordure de voies, soient dédiées aux nécropoles. Cet emplacement paraissait, de plus, favorable à la mise en place de cultures vivrières ; toutefois, les premières analyses polliniques et carpologiques n'ont pas été probantes sur ce sujet. Il semble que les thermes délimitent l'extension urbaine au nord, au départ des voies en direction de BordeauxBurdigala et de Limoges-Augustoritum. Quant à la partie ouest, elle accuse une forte déclivité avec des barres rocheuses présentant des à-pics interdisant toute urbanisation.

Les ultimes grands chantiers ont sans doute entraîné des changements de fonction de certains terrains. C'est notamment le cas avec la construction de l'amphithéâtre qui est installé, à proximité du forum, sur un terrain accusant une forte déclivité en direction de la rivière, au sud et à l'est. Il s'agit là d'un choix délibéré qui a permis une réelle économie de matériaux dans la mesure où la partie nord de l'édifice est encaissée dans le relief. La conséquence s'est traduite par l'apport, au-devant de la face sud et en fin de chantier, d'un remblai puissant de 3,5 $\mathrm{m}$ avec une forte proportion de rejets de déchets afin de niveler récentes ne sont pas accessibles.

2. La mention ancienne

en bordure de rivière,

du decumanus maximus,

pourrait correspondre

à des fragments rejetés. 


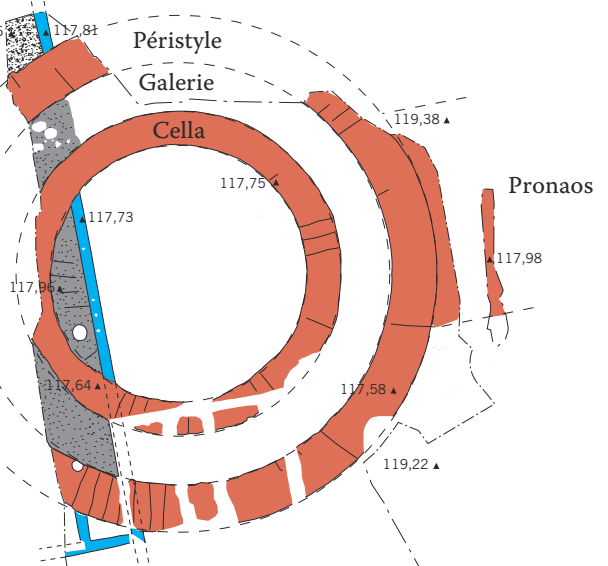

$\stackrel{\infty}{9}$

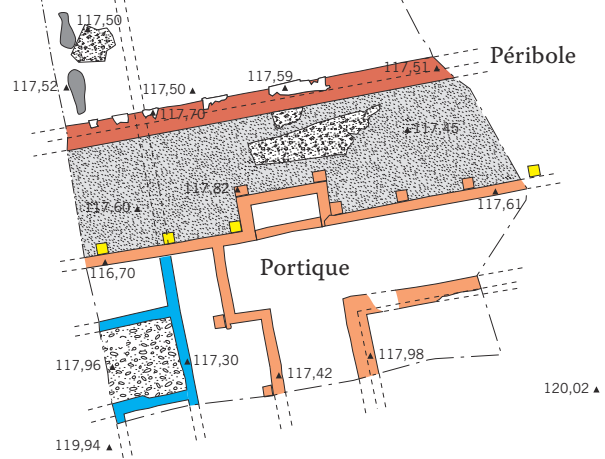

phase 1 : habitat conservé (20/50 $\mathrm{BC})$

phase 2 : portique ( $50 \mathrm{BC}$ )

phase 3 : temple (post. $50 \mathrm{BC}$ )

restitution des contreforts

voies/circulation

$\Lambda^{N}$

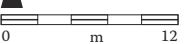

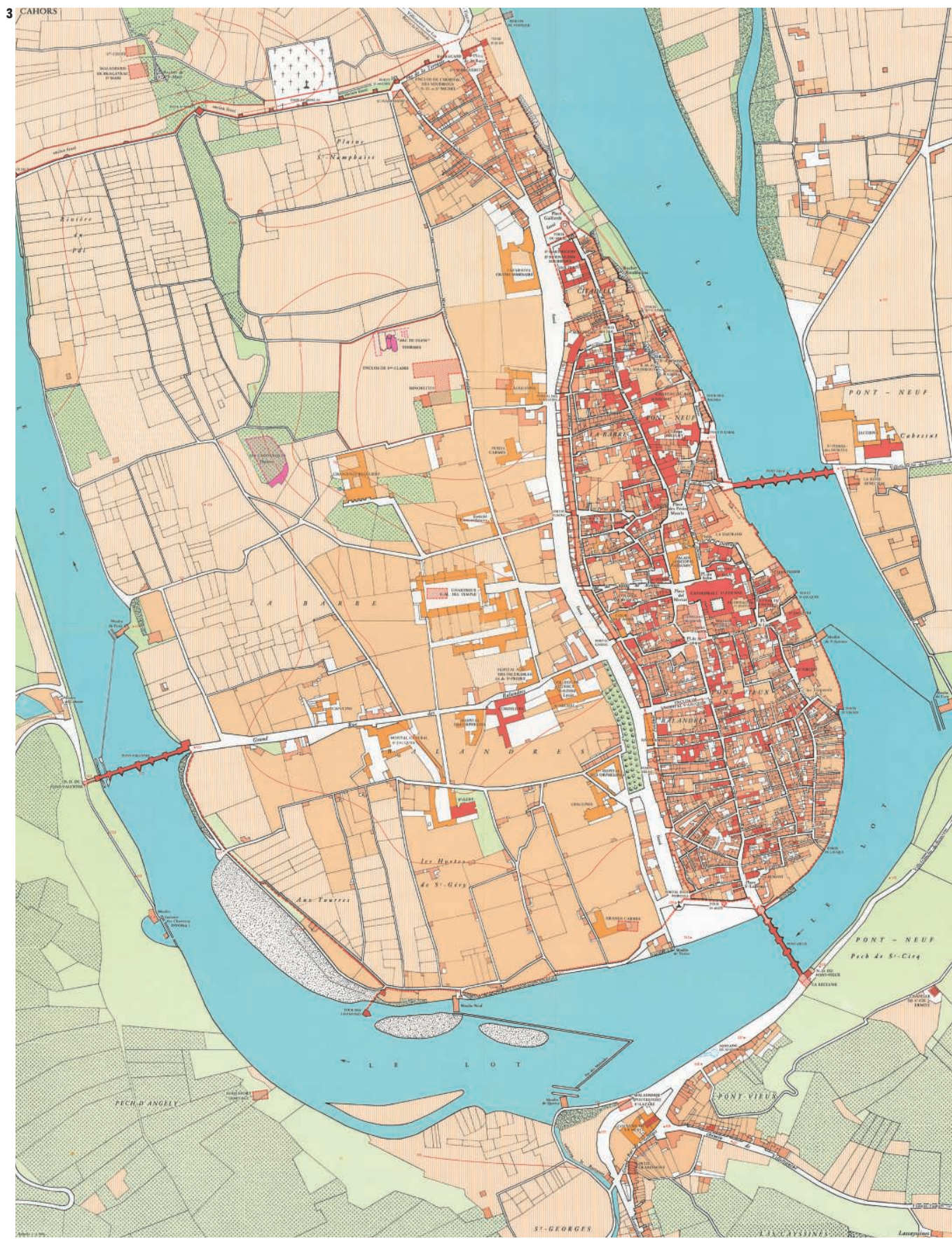

le pourtour du monument. Cet espace sera par la suite transformé en esplanade participant à la monumentalisation et offrant à la fois un lieu de passage et de rassemblement. On n'a pu déterminer si l'amphithéâtre avait été édifié sur une réserve foncière.

\section{Les espaces réservés}

Ils sont particulièrement difficiles à identifier, notamment à cause des bouleversements dus aux aléas fluviatiles sur les niveaux de sols. Mais dans le cas d'une ville antique construite $e x$-nihilo, on peut s'attendre à ce qu'elle suive une planification. Lors de la fouille préalable à l'extension du centre hospitalier, trois phases successives ont été identifiées [ill. 2]. Pour la première phase, dont n'est connue que la bordure est, sur une largeur de $5 \mathrm{~m}$, nous avons privilégié au moment de la fouille l'hypothèse d'un habitat, ce qui peut être maintenant remis en cause par la nouvelle perception que nous avons de l'alignement des monuments publics, mais sans qu'il soit possible de faire une nouvelle proposition plus étayée. La phase suivante correspond à un portique qui sera abandonné en raison de crues successives de la rivière, clairement perceptibles à la fouille avec des effets de battement de la nappe. C'est à la phase ultime, dans les années 60 de notre ère, qu'appartient le temple circulaire édifié sur un podium le mettant à l'abri des inondations. Cette construction correspond au transfert à l'intérieur de la ville du lieu de culte primitif fréquenté depuis
2. Temple de Cahors.

Phase 1: habitat; phase 2 . portique; phase 3 : temple de la divinité tutélaire. 3. Cartographie de Cahors sur fond cadastral ancien avec localisation des

principaux monuments antiques et médiévaux. 
la fin de l'indépendance. Sa construction était-elle prévue de longue date? Ou bien correspond-elle à une utilisation opportuniste d'un emplacement de faible densité de bâti ? Hormis une bande d'habitat sur sa bordure ouest, cet espace était dépourvu de tout aménagement antérieur et nous émettons l'hypothèse qu'il était en attente d'une construction prestigieuse ${ }^{\mathbf{3}}$.

La fouille partielle du forum n'a pas permis d'identifier de vestiges antiques qui lui soient antérieurs, ce qui incite à penser qu'il a été construit dans un espace réservé.

\section{Durant l'Antiquité tardive et le haut Moyen Âge}

Les observations réalisées aux thermes, au forum et sur l'amphithéâtre vont toutes dans le sens d'une désaffection de ces monuments dans la seconde moitié du IV ${ }^{\mathrm{e}}$ siècle et de la transformation de ces terrains en friches (Rigal, 2009). Les pièces d'apparat des bains sont subdivisées pour un usage d'habitat, le forum est démantelé et l'un des vomitoires de l'amphithéâtre est utilisé pour le parcage des animaux et comme lieu d'inhumation. Le cardo maximus perd sa fonction d'axe majeur de l'ordonnancement de la trame urbaine et ne subsiste plus que sous la forme d'une ruelle étroite, sinueuse et discontinue en raison d'aménagements privatifs qui empiètent progressivement sur la voirie. Un mausolée du Haut-Empire dominant la ville depuis le mont Saint-Cyr est également démantelé mais sa transformation répond à la volonté de créer un castellum sur cet emplacement hautement stratégique, en surplomb du pont et des routes du sud.

Il devient manifeste que la partie ouest de la ville est abandonnée au profit d'une restructuration de la partie est. De plus, même s'il n'existe pas de mentions historiques antérieures au VII ${ }^{\mathrm{e}}$ siècle, et si les traces archéologiques sont contemporaines des fortifications du XIII ${ }^{\mathrm{e}}$ siècle fermant le méandre au nord, ce nouvel espace urbain est protégé par une fortification dès l'Antiquité tardive, comme d'autres villescapitales romaines dont l'enceinte est connue. L'amphithéâtre conservait suffisamment d'élévation pour imposer une inflexion au rempart, tel que nous pouvons encore l'observer dans la trame urbaine de la ville qui présente une nette ondulation à l'emplacement restitué du petit côté est du monument. Cette ligne de rempart mettra à profit le premier cardo est, ce qui va lui donner son orientation rectiligne nord-sud, la partie orientale du rempart étant le long de la rivière.

Des incertitudes subsistent quant aux abords extérieurs du rempart. Dans cette ancienne zone urbaine, où les habitats sont abandonnés et dont les matériaux de construction sont récupérés, les indices d'occupation sont bien présents et révèlent des usages divers. Ces terrains servent à mener les animaux et à développer des pratiques culturales, usage qui aurait pu persister jusqu'au début du $\mathrm{XX}^{\mathrm{e}}$ siècle, où il est attesté, comme le suggère le toponyme les Hortes [ill. 3] (Rigal et al., 2011). On y dénombre aussi quelques sépultures isolées.

Il a été démontré que le lieu-dit les Hortes englobant une vaste superficie n'est plus urbanisé durant le Bas-Empire. La présence humaine y est néanmoins présente de façon assez soutenue jusqu'au début du ve siècle, puis de façon plus aléatoire jusqu'en l'an Mil, mais se pose là, pour pondérer ce constat, le problème de la reconnaissance du mobilier qui influe certainement sur notre réflexion. C'est à partir du XIII ${ }^{\mathrm{e}}$ siècle que des hôpitaux et de nombreux ordres mendiants (chartreux, capucins, cordeliers, chanoines réguliers, clarisses, petits et grands carmes, lazaristes, minorettes...) vont s'installer dans cette zone de cultures offrant de grands espaces disponibles, s'octroyant de vastes enclos abritant tout à la fois les édifices et les jardins (Rigal, Rousset, 2007).

C'est durant l'Antiquité tardive et surtout le haut Moyen Âge que le programme d'urbanisation de l'évêque Didier (reconstruction ou confortement de l'enceinte supposée du BasEmpire, édifices religieux, adduction...) s'inscrit en priorité dans la partie est du méandre, à l'abri du rempart délimitant un espace de 29 ha, mais également dans les Hortes avec le monastère dédié à saint Amans, future paroisse Saint-Géry. C'est ainsi que Divona, chef-lieu de la cité romaine, deviendra ville épiscopale au Moyen Âge. est l'égal de celui

de la cité voisine de

Périgueux-Vesunna.
Rigal D., 2008a, « Le temple de Cahors », in Monteil M. et Tranoy L. (dir.), La France gallo-romaine, Inrap, p. 132.

BoudARTChOUK J.-L., Rigal D., 2013, « Sites quercinois à vocation défensive, du Bas-Empire au haut Moyen Âge : un état des connaissances ", in ForssaC P. (éd.), Vivre et mourir en temps de guerre de la préhistoire à nos jours: Quercy et régions voisines, Actes du $59^{\mathrm{e}}$ Congrès d'études tenu à Cahors (19-21 juin 2009), Fédération Historique de Midi-Pyrénées - Société des Études du Lot, p. 27-51. Grimbert L., 2011, Cahors, Lot. 113 rue André Breton, rapport de diagnostic, Inrap-SRA Midi-Pyrénées.

Rigal D., 2004, "Le temple gallo-romain de Cahors ", in Bost J.-P. (dir.), Temples ronds monumentaux de la Gaule romaine, Journée d'étude, Bordeaux, 23 novembre 2003, Aquitania 20, p. 85-94.
Rigal D., 2008b, « La fouille des allées Fénelon à Cahors ", Annales des Rencontres Archéologiques de St Céré (Lot), 15, p. 95-98.

Rigal D., 2009, " Un regard nouveau sur Cahors-Divona, chef-lieu de la cité des Cadurques », Pallas, 79, Presses universitaires de Toulouse-le-Mirail, p. 377-399.

Rigal D., 2011, « Avatars et réaménagements de l'aqueduc antique de Cahors », in AbAdIE-REYNAL C., Provost S. et Vipard P. (ÉD.), Histoire des réseaux d'eau courante dans l'Antiquité. Réparations, modifications, réutilisations, abandons, récupération, Actes du colloque international, Nancy, 20-21 novembre 2009, Rennes, PUR, p. 47-61.
Rigal D., 2016, « La monumentalisation de CahorsDivona : nouveautés et acquis de la recherche récente ", in Bouet A. (dir.), Monumental!, la monumentalisation des villes de l'Aquitaine et l'Hispanie septentrionale durant le Haut-Empire, colloque de Villeneuve-sur-Lot, 10-12 septembre 2015, Aquitania, suppl. 37/1, p. 379-39o.

Rigal D. et al., 2017, Cahors (Lot), Allées Fénelon, rapport d'opération, Inrap-SRA Occitanie, 4 vol.

Rigal D. et al., 2011, "Cahors ", in Filippini A. et al., Carte archéologique de la Gaule (CAG-46), nouvelle édition avec compléments, les Belles Lettres, Paris, p. 93-143.

Rigal D., RousseT V., 2007, « La fouille archéologique des allées Fénelon à Cahors ", Bulletin de la Société des Études du Lot, 4, p. 301-309. 